\title{
Coexistence of orbital and CE-AFM orders in colossal magnetoresistance manganites: A symmetry perspective
}

\author{
J.L. Ribeiro \\ Centro e Departamento de Física da Universidade do Minho, Campus de Gualtar, 4710-057 Braga, Portugal
}

\section{A R T I C L E I N F O}

\section{Article history:}

Received 1 February 2016

Received in revised form

30 March 2016

Accepted 4 April 2016

Available online 4 April 2016

Keywords:

Magnetoresistance manganites

Symmetry and phase transitions

Ferromagnetism and anti-ferromagnetism

Orbital and charge order

\begin{abstract}
A B S T R A C T
The complex interplay between order parameters of different nature that dominates the physics of colossal magnetoresistance manganites is analysed from a symmetry based perspective. Phenomenological energies are given for the different competing phases. It is shown that the general trends observed in different systems, such as the mutual exclusion of orbital order and A-AFM order and the related stabilization of the CE-AFM order, stem to large extend from the symmetry of the parameters involved. The possible stabilization of complex phases where charge and orbital order coexist with magnetic and ferroelectric states is also anticipated.
\end{abstract}

(c) 2016 Elsevier B.V. All rights reserved.

\section{Introduction}

The phenomenon of colossal magnetoresistance in manganites is generally seen as a result of the competition between various order parameters of different nature (electronic, magnetic, and structural). Under certain conditions, different order parameters may arise in different temperature or field ranges, giving rise to well separate phases. That is the case, for instance, of the metallic ferromagnetic phases stabilized by a dominant double-exchange mechanism and strong Hund's rule coupling between mobile $e_{g}$ electrons and localized $t_{2 g}$ spins, or the antiferromagnetic insulator sates observed in most undoped systems, in which the dominant coupling occurs between the degenerate $e_{g}$ electrons and the Jahn-Teller distortions of $\mathrm{MnO}_{6}$ octahedra [1-4]. In many cases, however, several primary magnetic or structural order parameters may coexist in a single phase [5-7]. That is typically the case in the half-doped compounds $\mathrm{R}_{1-\mathrm{x}} \mathrm{A}_{\mathrm{x}} \mathrm{MnO}_{3}$ with $x=1 / 2$, (here $\mathrm{Re}$ is a 3 +rare earth metal ion and $A$ a 2 +alkaline earth metal ion), where the phase transitions observed may involve combined changes in magnetic, charge and orbital order states.

The mechanisms of coupling between several order parameters and its consequences are, in general, difficult to grasp directly from a microscopic level. The very notion of an order parameter stems from a macroscopic description [8], and it is closely related to the notion of symmetry and spontaneous symmetry breaking, Symmetry considerations may therefore provide useful frameworks for rationalizing the observed phase diagrams. A symmetry based

E-mail address: jlr@fisica.uminho.pt approach has the advantage of consider the system as a whole, taking into account all the relevant degrees of freedom (charge, occupational, structural or magnetic), and providing specific and exact forms of coupling between them. When taken in conjunction with the Landau theory of phase transitions (in which the transitions are described in terms of irreducible representations of a common parent phase), that approach may offer an integrate view of complex phase sequences. But, astonishingly, in the prolific literature on colossal magnetoresistance manganites, symmetry considerations are very seldom evoked.

In this work a pure phenomenological symmetry based approach is explored in order to draw the attention to the fact that many debated aspects of the physics of the manganites can be enlightened by simple symmetry considerations. The antipathy between ferromagnetism and orbital order, the reason why orbital order favors CE-AFM ground states, the connection between charge and orbital ordering, or why incommensurate charge order is expected above the Neel temperature at half-doping compounds [9-11], are examples of aspects that can be rationalized by following a very general phenomenological analysis that dispenses assumptions on specific microscopic mechanisms.

\section{The competing order parameters}

As in most perovskite systems, the tolerance factor plays an essential role in defining the nature of the critical modes that destabilize the orthorhombic Pnma parent phase of the $\mathrm{Re}_{1-x A x}$ $\mathrm{MnO}_{3}$ manganites. Typical end-members, such as $\mathrm{LaMnO}_{3}$ or $\mathrm{PrMnO}_{3}$ show insulating ground states with an A-type 
antiferromagnetic (AFM) order. The inclusion of a divalent ion, such as $\mathrm{Ca}^{2+}, \mathrm{Ba}^{2+}$ or $\mathrm{Sr}^{2+}$ [12-14], progressively induces additional instabilities and more complex phase transitions, which involve changes in magnetic, charge and orbital order, and, in some cases, metallicity. Although the details may vary from material to material, depending systematically on the sizes of the "A site" ions of the perovskite structure, some general trends can be identified.

Orbital order plays a pivotal role. The inclusion of divalent ions give rise to an imbalance of charge that must be accommodated by local changes of the valence of the $\mathrm{Mn}$ ions (from $\mathrm{Mn}^{3+}$ to $\mathrm{Mn}^{4+}$ ). For half-filling $(x=1 / 2)$, one could expect the existence of two well developed non-equivalent Mn sub-lattices, which may likely force the emergence of some kind of long-range order. That is precisely what happens in several half-filling systems, such as $\mathrm{La}_{1 / 2} \mathrm{Ca}_{1 / 2} \mathrm{MnO}_{3}, \mathrm{Pr}_{1 / 2} \mathrm{Ca}_{1 / 2} \mathrm{MnO}_{3}, \mathrm{Nd}_{1 / 2} \mathrm{Sr}_{1 / 2} \mathrm{MnO}_{3}, \mathrm{Nd}_{1 / 2} \mathrm{Ca}_{1 / 2} \mathrm{MnO}_{3}$, or $\mathrm{Pr}_{1 / 2} \mathrm{Sr}_{1 / 2} \mathrm{MnO}_{3}$ [15-23]. As first shown for the prototype case of $\mathrm{La}_{1 / 2} \mathrm{Ca}_{1 / 2} \mathrm{MnO}_{3}$ [15], the resulting structure corresponds to the doubling of the orthorhombic unit cell along $[1,0,0]$. The order parameter that emerges through charge doping corresponds therefore to a structural instability located at the $\mathrm{X}$-point $\left[\vec{k}=\left(\frac{1}{2}, 0,0\right)\right]$ of the Pnma Brillouin zone.

Since there are two 2-dim irreducible representations of the Pnma group at the X-point, there are two separate possibilities to consider (see Table 1). For an order parameter that transforms according to the irrep X1, for instance, the daughter phases may have the symmetries described by the space groups $P \frac{2_{1}}{m}$ if $\eta_{2}=0$, Pnm $2_{1}$ if $\eta_{1}=\eta_{2}$, or Pm otherwise (see Fig. 1). Here, $\eta_{1}$ and $\eta_{2}$ denote

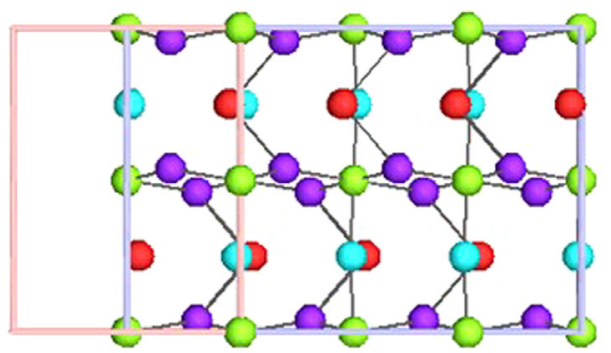

(a) Pnma

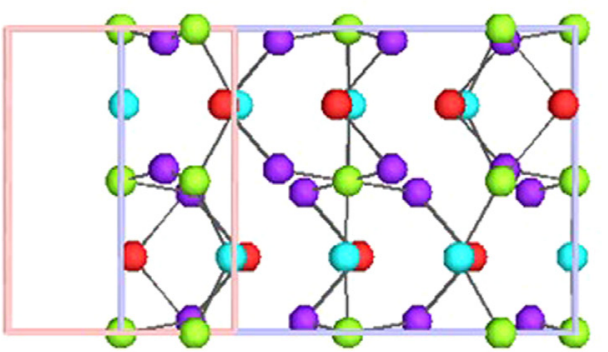

(b) $P 21 / m$

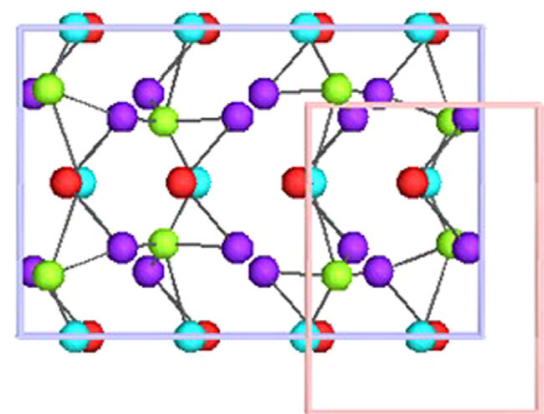

(c) Pnm21

Fig. 1. View along the [001] axis of the higher symmetry unit cells induced by an order parameter that transforms according to the irrep. X1 : (a) parent Pnma phase, (b) centrosymmetric P21/m $\left(\eta_{2}=0\right)$, and polar phase Pnm2 $2_{1}\left(\eta_{1}=\eta_{2}\right)$. For simplicity, only Mn displacements are shown. For a more detailed view see [24].
Table 1

Possible symmetries for the phases originated from different orientations of the 2-dim irreducible order parameters located at the X-point of the Brillouin zone; $\eta_{1}$ and $\eta_{2}$ denote the respective components in the real irreducible basis adopted by ISODISTORT [24]. For each case, the symmetry allowed secondary modes are also given.

\begin{tabular}{llll}
\hline Irrep & $\eta_{1} \neq 0 \quad \eta_{2}=0$ & $\eta_{1}=\eta_{2}$ & Otherwise \\
\hline $\mathrm{X} 1$ & $P \frac{2_{1}}{m}$ & $(\Gamma 2-)$ & $(\Gamma 4+, \Gamma 2-, \Gamma 3-)$ \\
& $(\Gamma 4+)$ & $P n a 2_{1}$ & $P c$ \\
$\mathrm{X} 2$ & $P \frac{2_{1}}{c}$ & $(\Gamma 2-)$ & $(\Gamma 4+, \Gamma 2-, \Gamma 3-)$ \\
& $(\Gamma 4+)$ & & \\
\hline
\end{tabular}

the two components of the order parameter defining its orientation in the irreducible linear space. A given set of symmetry allowed secondary modes can be ascribed to each case. For example, a scalar homogeneous order parameter $\zeta$ of symmetry $\Gamma 4+$ is allowed if $\eta_{2}=0$, or a polarization along the $z$-axis, $P_{z}$, transforming as $\Gamma 2-$, occurs if $\eta_{1}=\eta_{2}$. These secondary modes arise through the symmetry allowed mixed invariants $\zeta\left(\eta_{1}{ }^{2}-\eta_{2}{ }^{2}\right)$ and $\eta_{1} \eta_{2} P_{z}$, respectively. For a general orientation of the order parameter, a polarization along the $x$-axis, $P_{x}$ (transforming as $\Gamma 3-$ ) is also allowed through the higher order invariant $\eta_{1} \eta_{2}\left(\eta_{1}{ }^{2}-\eta_{2}{ }^{2}\right) P_{x}$.

It can be easily seen that the simple charge order pattern with alternating $\mathrm{Mn}^{3+}$ and $\mathrm{Mn}^{4+}$ first proposed by Goodenough [16] transforms exactly as $\Gamma 4+$ (see for instance [17]). One can therefore identify the allowed scalar mode, which we denote by $\zeta$, with this charge order process. That is, the charge order is here a secondary effect of the orbital order, driven by the symmetry allowed coupling invariant $\zeta\left(\eta_{1}{ }^{2}-\eta_{2}{ }^{2}\right){ }^{1}$ Notice that the charge order disappears if $\eta_{1}=\eta_{2}$ and the polar group $P n m 2_{1}$ is realized, but it may coexist with a ferroelectric polarization in the $x z$-plane if the orbital order parameter condenses along an arbitrary direction, and the symmetry of the phase is reduced to $\mathrm{Pm}$. More complex scenarios of this type have been suggested [25] (in which bondcentered polarons could coexist with site centered charge order, leading to the breaking of inversion and to the induction of polar states). In this context, $\operatorname{Pr}_{1-x} \mathrm{Ca}_{x} \mathrm{MnO}_{3}$ has been referred to as a new paradigm for ferroelectrics [26-28]. But so far, no clear direct proof of the existence of an electric polarization could be obtained $[29,30]$.

The simple $\mathrm{Mn}^{3+} / \mathrm{Mn}^{4+}$ charge ordering described by the $\Gamma 4+$ mode may not be realized in all half-filled compounds [21$23,25]$. Quite often $[6,19,31]$ large JahnTeller distortions of the oxygen octahedra surrounding both sites of inequivalent $\mathrm{Mn}$ ions are observed, and the valence contrast between the sub-lattices is reduced to $3.5+\delta$ and $3.5-\delta$, with $\delta$ not quite close to 0.5 . Here, the $e_{g}$ electron could be partly shared by two $\mathrm{Mn}^{3+}$, forming a dimer like state known as a bond-centered Zener polaron [22]. Moreover, the valence contrast could be spatial modulated giving rise to an incommensurate charge density wave. Such a scenario is, in fact, quite expectable, since a Lifshitz invariant $\left[\eta_{1} \partial \eta_{2} / \partial x-\eta_{2} \partial \eta_{1} / \partial x\right]$ is allowed by symmetry at the X-point of the Brillouin zone. As a result, an intermediate incommensurate phase sandwiched between the paramagnetic Pnma1' and the commensurate orbital ordered phase must occur within some temperature range. In $\mathrm{Pr}_{0.5} \mathrm{Ca}_{0.5} \mathrm{MnO}_{3}$, for example, such an intermediate phase is observed, with a temperature dependent

\footnotetext{
${ }^{1}$ The von Neumann's principle (a secondary mode must comply with the symmetry breaking imposed by the primary order parameter) allows us to establish a hierarchy between different order parameters. Here, the translational symmetry group induced by the orbital ordering parameter is a sub-group of the translational symmetry imposed by the charge order alone. Therefore, it is the former the primary driven mode.
} 
incommensurate structural wave vector $\vec{k}_{i n c}=\left(\frac{1}{2}-\varepsilon, 0,0\right)$. Given the relationship

discussed above between the charge and the orbital ordering processes, a secondary incommensurate charge density wave characterized by a wave number $2 \vec{k}_{\text {inc }}=2 \varepsilon$ is also to be expected. ${ }^{2}$ Eventually, in some compounds, the lock-in at the X-point of the Brillouin zone may not occur, and the orbital and charge order remain incommensurate, or the temperature range of stability of the incommensurate phase may be too narrow to be detected. But, the description of the orbital and charge order processes, either commensurate or incommensurate, requires only two separate parameters, one structural and one scalar, located at the points $\vec{k}=\left(\frac{1}{2}, 0,0\right)$ and $\vec{k}=(0,0,0)$ of the Brillouin zone, respectively.

Let us consider now the magnetic order parameters. As seen, the progressive onset of the orbital order state is accompanied by a modification of the magnetic ground state. Near half doping, the lowest temperature phase is typically insulating, with simultaneous charge, orbital and CE type antiferromagnetic order. The charge/orbital ordering process that sets in at higher temperatures plays here a crucial role because it is indispensable to the formation of the new magnetic state.

As shown in Fig. 2, the new CE-type antiferromagnetic order can be viewed as a set of ferromagnetic zig-zag chains placed in the ac-plane, antiferromagnetically ordered relative to each other, with a 4-fold magnetic cell (when referred to the prototype orthorhombic structure). The multiple magnetic cell of the novel phase reflects the condensation of a 2-dim primary magnetic order parameter of symmetry $m U 1+U 4+\left(\right.$ see $\left.^{3}\right)$, with components $\left(\rho_{1}, \rho_{2}\right)$, and a wave vector $\vec{k}=\left(\frac{1}{2}, 0, \frac{1}{2}\right)$, superposed to a previously established centrosymmetric commensurate orbital order $P \frac{21}{m}$ (that is, driven by a structural mode $\mathrm{X} 1$, see Table 1 ). ${ }^{4}$ The commensurate orbital order is essential to ensure both the existence of two non-equivalent Mn sub-lattices, and the onset of a secondary 2-dim magnetic mode of symmetry mZ1 at $\vec{k}=\left(0,0, \frac{1}{2}\right)$, which arises through the mixed invariants $\eta_{1} \rho_{1} z_{1}+\eta_{2} \rho_{2} z_{2}$ and $\eta_{1} \rho_{2} z_{1}-\eta_{2} \rho_{1} z_{2}$ (with $z_{1}$ and $z_{2}$ denoting the components of the secondary magnetic mode). Such a mode represents in fact an essential ingredient of the new spin configuration, because the CE-AFM order requires a superposition of both modes, each dominating the spin configuration in separate sublattices: the $m U 1+U 4+$ mode mainly defining the configuration of the $\mathrm{Mn}^{4+}$ corner sites sub-lattice, and the secondary $m Z 1$ mode mainly affecting the spin configuration of the $\mathrm{Mn}^{3+}$ bridge-sites sub-lattice $[6,24,31]$. The unusual prominence of the secondary magnetic mode in this latter sub-lattice is consistent with the large Jahn-Teller distortions that the orbital ordering process induces in the $\mathrm{Mn}^{3+} \mathrm{O}_{6}$ octahedra (in contrast with the case of the $\mathrm{Mn}^{4+} \mathrm{O}_{6}$ octhedra, which remain almost undistorted) [31]. Notice that in spite of the different dominant propagation vectors, the spin orders in the two sub-lattices are not independent of each other. Both sub-lattices order at the same

\footnotetext{
${ }^{2}$ Since such an incommensurate order cannot be described by an ordinary space group, even for a small $\varepsilon$, the refinements of the diffraction data obtained by using constraints imposed by the space group necessarily lead to reduced average charge contrasts between the two manganese sub-lattices. It would be interesting, in this respect, to re-analyse the diffraction data of the so-called "incomplete charge ordered phase" by considering proper symmetry constraints provided by the superspace formalism, namely by assuming the expected symmetry .

${ }^{3}$ The prefix $m$ means that the irrep is odd under time reversal, as required for a magnetic order parameter.

${ }^{4}$ In some compounds, the lock-in of the orbital order at the X-point of the Brillouin zone triggers the onset of the CE-type AFM order. In other compounds, the lock-in of the orbital order at $T_{\mathrm{L}}$ and the onset of the magnetic phase at $T_{\mathrm{N}}$ occurs at separate temperatures, but with $T_{\mathrm{N}}<T_{\mathrm{L}}$. The orbital order is here an essential ingredient of the magnetic order.
}

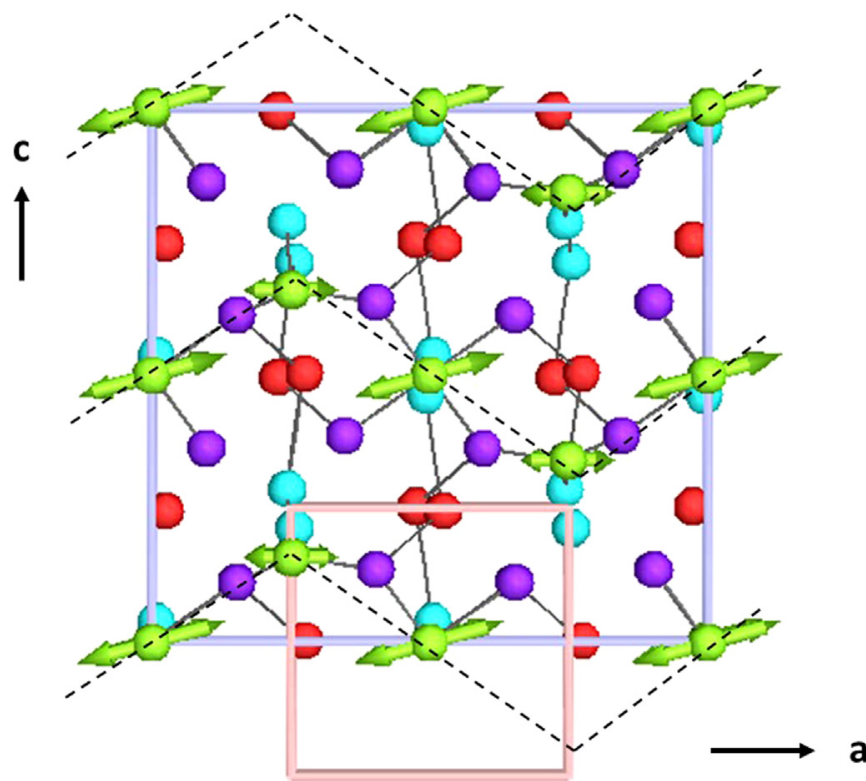

Fig. 2. View of the unit cell of the CE-AFM phase along [010]. This antiferromagnetic order coexists with charge and orbital order. Different average spins can be ascribed to the two non-equivalent magnetic Mn sub-latices. For easier identification, the "bridge sites" spins are represented by longer arrows, slightly tilted towards the $c$-axis, while the "corner sites" spins are represented by shorter arrows strictly directed along the $a$-axis. The mZ1 mode and the $m U 1+U 4+$ mode dominate the spin configuration in the bridge sites and in the corner sites, respectively. The dashed lines identify the ferromagnetic zig-zag chains of spins that are characteristic of this phase. For clarity, all the atomic displacements and strain modes allowed by symmetry were erased, with the exception of the displacements of the $\mathrm{Mn}^{4+}$ ions located at the corner sites (which were assumed to be directed along the $c$-axis, as it is observed in $\mathrm{La}_{1 / 2} \mathrm{Ca}_{1 / 2} \mathrm{MnO}_{3}$ [31]). For a more detailed view of the structure see [24].

critical temperature due to the condensation of a single magnetic primary mode (the $m U 1+U 4+$ mode) superposed to a previously established orbital order, and the spin orientations in the two sub-lattices are correlated in such a way that the one dimensional ferromagnetic zig-zag chains, characteristic of the CEAFM order, are formed (see Fig. 2).

\section{How orbital order changes the magnetic ground state}

The Landau free energy Ansatz related to the condensation of the different order parameters described in the section above can be straightforwardly obtained, once their transformation properties are known. Since the role of the secondary modes amounts to a mere renormalization of the coefficients of the Landau free energy, only functionals expressed in terms of the primary order parameters will be considered. It will be shown that the orbital and charge order state is not only an essential ingredient of the novel magnetic ground state, but also that it may actually trigger the condensation of the magnetic $m U 1+U 4+$ mode, suppressing at the same time the magnetic instability at the center of the Brillouin zone.

The free energy of the A-type magnetic order parameter is rather trivial, since all the magnetic irreducible representations of the grey group Pnma1' at the $\Gamma$-point are one dimensional. The free energy for this phase is:

$G_{1}=\frac{1}{2} a\left(T-T_{c}\right) M^{2}+\frac{1}{4} b M^{4}$

We have denoted the active parameter by $M$ (which may correspond for instance to the ${ }_{A x}$-type AFM order), and ignored the contribution of the corresponding symmetry allowed pseudo 
proper parameters ( $F_{y}$ or $G_{z}$ modes, in that case). For convenience, we will define reduced free energy and temperature scales $F_{1}=\frac{G}{b}$, and $t=\frac{a\left(T-T_{\mathrm{c}}\right)}{\mathrm{b}}$, in which case (1) becomes $F_{1}=\frac{1}{2} t M^{2}+\frac{1}{4} M^{4}$. In equilibrium, we have then $M^{2}=-t$ and $F_{1}=-\frac{t^{2}}{4}$.

For the competing magnetic order parameter (whose components transform, as seen, according to the 2-dim physically irreducible representation $m U 1+U 4+$ ), the symmetry allowed invariants up to the fourth order give the following energy expansion:

$F_{2}=\frac{1}{2}\left(t+\delta_{1}\right)\left(\rho_{1}^{2}+\rho_{2}^{2}\right)+\frac{1}{4} b_{2}\left(\rho_{1}^{4}+\rho_{2}^{4}\right)+\frac{1}{2} c_{2} \rho_{1}^{2} \rho_{2}^{2}$

$$
+\frac{1}{2} d_{2} \rho_{1} \rho_{2}\left(\rho_{1}^{2}-\rho_{2}^{2}\right)
$$

Here, we have adopted the same reduced energy and temperature scales. The parameter $\delta_{1}$ defines, in that scale, the temperature at which the quadratic term changes its sign. A $\delta_{1}>0$ (or $<0$ ) means that the corresponding magnetic instability occurs at a lower (or higher) temperature than that of $M$, which we take as reference.

The free energy density related with the orbital order parameter $\left(\eta_{1}, \eta_{2}\right)$ is far more complex. It can be written as:

$$
\begin{aligned}
f_{3}= & \frac{1}{2}\left(t+\delta_{2}\right)\left(\eta_{1}^{2}+\eta_{2}^{2}\right)+\frac{1}{4} b_{3}\left(\eta_{1}^{2}+\eta_{2}^{2}\right)^{2}+\frac{1}{2} c_{3} \eta_{1}^{2} \eta_{2}^{2} \\
& +\frac{1}{2} d_{3}\left(\eta_{1} \frac{\partial \eta_{2}}{\partial x}-\eta_{2} \frac{\partial \eta_{1}}{\partial x}\right)+e\left(\left(\frac{\partial \eta_{1}}{\partial x}\right)^{2}+\left(\frac{\partial \eta_{2}}{\partial x}\right)^{2}\right)
\end{aligned}
$$

We have collected again invariant terms up to the fourth order, including the Lifshitz invariant responsible for the stabilization of the incommensurate phase. Notice that the relative critical temperature $\delta_{2}$ is expected to be strongly dependent on the charge filling $x$, since the orbital ordering is induced by doping. Finally, we have to consider the coupling energy for the three order parameters, which we take limited to the possible fourth order mixed invariants:

$$
\begin{aligned}
f_{\text {int }}= & \Delta_{1}\left(\rho_{1}^{2} \eta_{1}^{2}+\rho_{2}^{2} \eta_{2}^{2}\right)+\Delta_{2}\left(\rho_{1}^{2} \eta_{2}^{2}+\rho_{2}^{2} \eta_{1}^{2}\right)+\Delta_{3}\left(\eta_{1}^{2}-\eta_{2}^{2}\right) \rho_{1} \rho_{2} \\
& +\Delta_{4}\left(\eta_{1}^{2}+\eta_{2}^{2}\right) M^{2}+\Delta_{5}\left(\rho_{1}^{2}+\rho_{2}^{2}\right) M^{2}
\end{aligned}
$$

Let us now briefly see how the three main phases discussed above (the incommensurate orbital order and the two higher symmetry commensurate states listed in Table 1) can emerge from the energy $F=F_{1}+F_{2}+\frac{1}{L} \int\left[f_{3}(x)+f_{\text {int }}(x)\right] d x$, where $L$ is the modulation wavelength. Consider first the case of an incommensurate phase. Here the components of the order parameter $\eta$ are spatially modulated, and the couplings with the polarization and homogeneous charge order are forbidden due to the breaking of translational invariance. The symmetry of the resulting incommensurate phase is uniquely determined by the symmetry of the order parameter, and is described by the centrosymmetric superspace group Pnma1' $(\alpha, 0,0)$ s0ss (or $\operatorname{Pmcn}^{\prime}(0,0, \gamma) 000$ in the ISODISTORT [24] setting).

In general, the minimization of the energy of the incommensurate phase must follow the usual methodology (see for instance [32]). However, for the present purpose, one can adopt the simplified path of assuming that the modulation is strictly harmonic, and expressed by the trial solutions $\eta_{1}=\eta \cos [k x+\varphi]$ and $\eta_{2}=\eta \sin [k x+\varphi]$. Here, $k$ is the modulation wave vector seen from the X-point (i.e., the actual wave vector is $q=\frac{1}{2}-k$, so that the lock-in corresponds to the homogeneous phase $k=0$ ). In this approximation, the energy density becomes:

$$
\begin{aligned}
f_{\text {inc }}(x)= & \frac{1}{2} t M^{2}+\frac{1}{4} M^{4}+\frac{1}{2}\left(t+\delta_{1}\right) \eta^{2}+\frac{1}{4} b \eta^{4} \\
& +c \eta^{4} \cos ^{2}[k x+\varphi] \sin ^{2}[k x+\varphi]+d k \eta^{2}+e k^{2} \eta^{2}+ \\
& +\frac{1}{2}\left(t+\delta_{2}\right)\left(\rho_{1}^{2}+\rho_{2}^{2}\right)+\frac{1}{4} b_{3}\left(\rho_{1}^{4}+\rho_{2}^{4}\right)+\frac{1}{2} c_{3} \rho_{1}^{2} \rho_{2}^{2} \\
& +\frac{1}{2} d_{3} \rho_{1} \rho_{2}\left(\rho_{1}^{2}-\rho_{2}^{2}\right)+ \\
& +\Delta_{1} \eta^{2}\left(\rho_{1}^{2} \cos ^{2}[k x+\varphi]+\rho_{2}^{2} \sin ^{2}[k x+\varphi]\right) \\
& +\Delta_{2} \eta^{2}\left(\rho_{1}^{2} \sin ^{2}[k x+\varphi]+\rho_{2}^{2} \cos ^{2}[k x+\varphi]\right)+ \\
& +\Delta_{3} \eta^{2} \rho_{1} \rho_{2}\left(\cos ^{2}[k x+\varphi]-\sin ^{2}[k x+\varphi]\right)+\Delta_{4} \eta^{2} M^{2} \\
& +\Delta_{5}\left(\rho_{1}^{2}+\rho_{2}^{2}\right) M^{2}
\end{aligned}
$$

After integration over $L$, the $c$-and the $\Delta_{3}$ - terms average out, leading to the energy:

$$
\begin{aligned}
F_{\text {inc }}= & \frac{1}{2}\left(t+2 \Delta_{4} \eta^{2}\right) M^{2}+\frac{1}{4} M^{4}+\frac{1}{2}\left(t+\delta_{1}+d k+e k^{2}\right) \eta^{2}+\frac{1}{4} b \eta^{4}+ \\
& +\frac{1}{2}\left(t+\delta_{2}+\left(\Delta_{1}+\Delta_{2}\right) \eta^{2}\right)\left(\rho_{1}^{2}+\rho_{2}^{2}\right)+\frac{1}{4} b_{3}\left(\rho_{1}^{4}+\rho_{2}^{4}\right) \\
& +\frac{1}{2} c_{3} \rho_{1}^{2} \rho_{2}^{2}+\frac{1}{2} d_{3} \rho_{1} \rho_{2}\left(\rho_{1}^{2}-\rho_{2}^{2}\right)+\Delta_{5}\left(\rho_{1}^{2}+\rho_{2}^{2}\right) M^{2}
\end{aligned}
$$

The transition to the incommensurate phase occurs when the quadratic term in $\eta$ vanishes, with $k$ real. Therefore, by imposing $t+\delta_{1}+d k+e k^{2}=0$, one obtains $k_{ \pm}=\frac{-d \pm \sqrt{d^{2}-4 e\left(t+\delta_{1}\right)}}{2 e}, t_{i}=\frac{d^{2}}{4 e}-\delta_{1}$, and $k\left(t_{i}\right)=-\frac{d}{2 e}$. The free energy of the incommensurate phase can then be expressed as:

$$
\begin{aligned}
F_{\text {inc }}= & \frac{1}{2}\left(t+2 \Delta_{4} \eta^{2}\right) M^{2}+\frac{1}{4} M^{4}+\frac{1}{2}\left(t+\delta_{1}-\frac{d^{2}}{4 e}\right) \eta^{2}+\frac{1}{4} b \eta^{4}+ \\
& +\frac{1}{2}\left(t+\delta_{2}+\left(\Delta_{1}+\Delta_{2}\right) \eta^{2}\right)\left(\rho_{1}^{2}+\rho_{2}^{2}\right)+\frac{1}{4} b_{3}\left(\rho_{1}^{4}+\rho_{2}^{4}\right) \\
& +\frac{1}{2} c_{3} \rho_{1}^{2} \rho_{2}^{2}+\frac{1}{2} d_{3} \rho_{1} \rho_{2}\left(\rho_{1}^{2}-\rho_{2}^{2}\right)+\Delta_{5}\left(\rho_{1}^{2}+\rho_{2}^{2}\right) M^{2}
\end{aligned}
$$

Here, we have assumed (again for simplicity sake) that the modulation wave length varies very little with the temperature, so that $k \approx k\left(t_{i}\right)$ remains a good approximation for $t<t_{i}$. An important conclusion that can be drawn from the above expression is that the onset of an incommensurate orbital order destabilizes both magnetic order parameters $M$ and $\left(\rho_{1}, \rho_{2}\right)$ (by decreasing their corresponding effective critical temperatures), if the coupling constants $\Delta_{1}, \Delta_{2}$, and $\Delta_{4}$ are positive. This conclusion is independent of the simplifying assumptions made.

Let us consider now the two higher symmetry commensurate solutions, with $k=0$. One of these corresponds to the commensurate orbital order with the polar symmetry Pnm $2_{1} 1^{\prime}$, induced by a lock-in at the X-point with $\varphi=\frac{\pi}{4}(\bmod . \pi)$, fixing the order parameter orientation $\eta_{1}=\eta_{2}=\frac{\eta}{\sqrt{2}}$. The corresponding free energy $F_{a}$ is:

$$
\begin{aligned}
F_{a}= & \frac{1}{2}\left(t+2 \Delta_{4} \eta^{2}\right) M^{2}+\frac{1}{4} M^{4}+\frac{1}{2}\left(t+\delta_{1}\right) \eta^{2}+\frac{1}{4}\left(b+\frac{1}{2} c\right) \eta^{4} \\
& +\frac{1}{2}\left(t+\delta_{2}+\left(\Delta_{1}+\Delta_{2}\right) \eta^{2}\right)\left(\rho_{1}^{2}+\rho_{2}^{2}\right)+ \\
& +\frac{1}{4} b_{3}\left(\rho_{1}^{4}+\rho_{2}^{4}\right)+\frac{1}{2} c_{3} \rho_{1}^{2} \rho_{2}^{2}+\frac{1}{2} d_{3} \rho_{1} \rho_{2}\left(\rho_{1}^{2}-\rho_{2}^{2}\right) \\
& +\Delta_{5}\left(\rho_{1}^{2}+\rho_{2}^{2}\right) M^{2}
\end{aligned}
$$

Once again, the same conclusion is achieved. The onset of the polar phase hinders the magnetic phases, if the coupling constants $\Delta_{1}, \Delta_{2}$, and $\Delta_{4}$ are positive. In such a case, magnetism and ferroelectricity tend to avoid each other.

The commensurate orbital and charge ordered state with symmetry $P \frac{21}{m} 1^{\prime}$ corresponds to the choice $\varphi=0(\bmod . \pi)$ and $\eta_{2}=0$. For this orientation of the order parameter, the free energy becomes: 


$$
\begin{aligned}
F_{b}= & \frac{1}{2}\left(t+2 \Delta_{4} \eta_{1}^{2}\right) M^{2}+\frac{1}{4} M^{4}+\frac{1}{2}\left(t+\delta_{1}\right) \eta_{1}^{2}+\frac{1}{4} b \eta_{1}^{4} \\
& +\frac{1}{2}\left(t+\delta_{2}\right)\left(\rho_{1}^{2}+\rho_{2}^{2}\right)+\frac{1}{4} b_{3}\left(\rho_{1}^{4}+\rho_{2}^{4}\right)+ \\
& +\frac{1}{2} c_{3} \rho_{1}^{2} \rho_{2}^{2}+\frac{1}{2} d_{3} \rho_{1} \rho_{2}\left(\rho_{1}^{2}-\rho_{2}^{2}\right)+\left(\Delta_{1} \rho_{1}^{2}+\Delta_{2} \rho_{2}^{2}\right) \eta_{1}^{2} \\
& +\Delta_{3} \eta_{1}^{2} \rho_{1} \rho_{2}+\Delta_{5}\left(\rho_{1}^{2}+\rho_{2}^{2}\right) M^{2}
\end{aligned}
$$

Here, as before, the centrosymmetric orbital order destabilizes the homogeneous magnetic order parameter M. But the coupling with the $m U 1+U 4+$ mode is now different. In particular, a new coupling term $\Delta_{3} \eta_{1}^{2} \rho_{1} \rho_{2}$ arises, expressing the symmetry compatibility between the centrosymmetric orbital state $\left(P \frac{2_{1}}{m} 1^{\prime}\right)$ and the phase that this parameter would stabilize by itself. In other words, in the absence of orbital ordering (i.e., in the case of a direct transition from the parent phase to the AFM phase driven by the $m U 1+U 4+$ mode), the magnetic order parameter would yield a spin arrangement with symmetry $P_{a} \frac{2_{1}}{m}$ (see ${ }^{5}$ ), and the charge ordered state described by the same parameter $\xi$ [which is also induced by the centrosymmetric orbital order; here, $\xi$ originates from the mixed invariants $\xi\left(\lambda_{1} \rho_{1} \rho_{2}+\lambda_{2}\left(\rho_{1}^{2}-\rho_{2}^{2}\right)\right]$. This symmetry compatibility $\left(P_{a} \frac{2_{1}}{m} \subset P \frac{2_{1}}{m} 1^{\prime}\right)$ is expressed by the existence of an additional "attractive" interaction term between the two order parameters, which has the form $-\gamma \eta_{1}^{2}\left(\lambda_{1} \rho_{1} \rho_{2}+\lambda_{2}\left(\rho_{1}^{2}-\rho_{2}^{2}\right)\right)$. Here, $\gamma$, $\lambda_{1}$, and $\lambda_{2}$ are coupling constants that we take as positive. In particular, the coefficient $\Delta_{3}$ in (9) is lowered to $\Delta_{3}-\gamma \lambda_{1}$. Moreover, the secondary magnetic mode $\mathrm{mZ1}$, with $\vec{k}=\left(0,0, \frac{1}{2}\right)$, also has a similar effect. Since this mode originates from the trilinear invariants $\lambda_{3}\left(\eta_{1} \rho_{1} z_{1}+\eta_{2} \rho_{2} z_{2}\right)$ and $\lambda_{4}\left(\eta_{1} \rho_{2} z_{1}-\eta_{2} \rho_{1} z_{2}\right)$, its net effect is to reinforce a negative effective coupling through the additional term $-\lambda_{3} \lambda_{4} \eta_{1}^{2} \rho_{1} \rho_{2}$. As a result the coefficient $\Delta_{3}$ that gauges the intensity of the coupling term specific to this particular orientation of the order parameter is modified to $\Delta_{3}-\gamma \lambda_{1}-\lambda_{3} \lambda_{4}$, and may become negative.

Let us now see that, for an effective $\Delta_{3}<0$ in (9), the lock-in of the orbital order into the centrosymmetric commensurate phase may suffice to trigger (or at least to favor) the condensation of the $m U 1+U 4+$ mode, stabilising in consequence the CE-AFM spin arrangement. This can be seen in the following way. In equilibrium, the components of the magnetic mode are found by imposing the conditions $\frac{\partial F_{b}}{\partial \rho_{1}}=\frac{\partial F_{b}}{\partial \rho_{2}}=0$. Since close to the magnetic transition $\rho_{1(2)}<<1$, one can linearize the resulting equations. The equilibrium condition lead then to the homogeneous system:

$\left[\begin{array}{cc}t+\delta_{2}+2 \Delta_{5} M^{2}+2 \Delta_{1} \eta_{1}^{2} & \Delta_{3} \eta_{1}^{2} \\ \Delta_{3} \eta_{1}^{2} & t+\delta_{2}+2 \Delta_{5} M^{2}+2 \Delta_{2} \eta_{1}^{2}\end{array}\right] \times\left[\begin{array}{l}\rho_{1} \\ \rho_{2}\end{array}\right]=0$

Therefore, either the system remains paramagnetic with $\rho_{1}=\rho_{2}=0$, or det $\left[\begin{array}{cc}t+\delta_{2}+2 \Delta_{5} M^{2}+2 \Delta_{1} \eta_{1}^{2} & \Delta_{3} \eta_{1}^{2} \\ \Delta_{3} \eta_{1}^{2} & t+\delta_{2}+2 \Delta_{5} M^{2}+2 \Delta_{2} \eta_{1}^{2}\end{array}\right]=0$ if $\rho_{1}$ and $\rho_{2}$ become finite. That is, the magnetic order becomes possible at $t=t_{N}$, providing that:

$$
\begin{aligned}
\left(t_{N}+\right. & \left.\delta_{2}+2 \Delta_{5} M^{2}\right)^{2}+2\left(\Delta_{1}+\Delta_{2}\right)\left(t_{N}+\delta_{2}+2 \Delta_{5} M^{2}\right) \eta_{1}^{2} \\
& +\left(4 \Delta_{1} \Delta_{2}-\Delta_{3}^{2}\right) \eta_{1}^{4}=0
\end{aligned}
$$

In the absence of orbital order, $\eta_{1}=0$, the condensation of the magnetic parameter would occur at $t_{N}=-\left(\delta_{2}+2 \Delta_{5} M^{2}\right)$. However, if the effective coupling $\Delta_{3}$ is strong enough to impose a negative quartic term (i.e., if $4 \Delta_{1} \Delta_{2}<\Delta_{3}^{2}$ ) that dominates over the positive

\footnotetext{
${ }^{5}$ The subscript a in the label of the magnetic group means that the time reversal operation of the grey group $\mathrm{P} 12_{1} / \mathrm{m} 11^{\prime},\left\{\begin{array}{llll}1^{\prime} & 0 & 0 & 0\end{array}\right\}$ is replaced by time reversal combined with a shift of $\pi$ of the magnetic modulation, $\left\{1^{\prime} \mid 1 / 200\right\}$.
}

quadratic term, then the transition temperature can be shifted towards a higher value by

$$
\Delta t_{N}=\left(\Delta_{1}+\Delta_{2}\right) \eta_{1}^{2}\left[\sqrt{1+\frac{\Delta_{3}^{2}-4 \Delta_{1} \Delta_{2}}{\left(\Delta_{1}+\Delta_{2}\right)^{2}}}-1\right] \text {. In other words, the pre- }
$$
sence of a centrosymmetric orbital order accompanied by a full charge order state may favor the condensation of the $m U 1+U 4+$ mode and the stabilization of the CE-AFM phase, in detriment of the homogeneous magnetic order that prevails in the undoped systems.

\section{Conclusion}

The phase diagrams in many manganites reveals complex phases where at least three primary order parameters (two magnetic and one structural) compete. The identification of the symmetry of these primary parameters allows one to establish a unified picture for the phase transitions observed, and to characterize the different secondary modes that may arise as secondary effects. In particular, charge order must be seen as a secondary effect of the onset of a centrosymmetric orbital order state. Hence, while a centrosymmetric orbital order induces a full charge order state, an incommensurate orbital order generates an incommensurate charge density wave. The conditions under which the same structural instability may yield a ferroelectric state were also characterized, along with the possibility of coexistence in complex phases of charge, orbital order and ferroelectricity. The symmetries of the different possible phases, either commensurate or incommensurate, were anticipated (and therefore their corresponding ferroic properties fully specified), making possible a direct contact with experimental results.

The characterization of the coupling between the structural mode (induced by charge doping) with the two competing primary magnetic modes has been made within a phenomenological Ginzburg-Landau theory. The form of that coupling allowed us to rationalize the progressive destabilization the A-AFM order in favor of the CE-AFM spin arrangement, in terms of the symmetry, whenever a centrosymmetric orbital order is established by charge doping. This main feature of the observed phase diagrams can therefore be viewed as an expected result of the symmetry of the parameters involved.

\section{Acknowledgments}

This work was supported by the Portuguese Foundation for Science and Technology (FCT) in the framework of the Strategic Projects of CFUM [PEst-C/FIS/UI0607/2013 (F-COMP-01-0124FEDER022711)].

\section{References}

[1] M.B. Salamon, M. Jamie, The physics of manganites: structure and transport, Rev. Mod. Phys. 76 (2001) 583.

[2] Y. Tokura (Ed.), Colossal Magnetoresistance Oxides, Gordon \& Breach, New York, 2000.

[3] E. Dagotto, T. Hotta, A. Moreo, Phys. Rep. 344 (2001) 1.

[4] E. Dagotto, Nanoscale Phase Separation and Colossal Magnetoresistance, Springer-Verlag, Berlin, 2002.

[5] V.N. Loktev, Yu. G. Pogorelov, Low Temp. Phys. 26 (2000) 171.

[6] O. Cépas, H.R. Krishnamurthy, T.V. Ramakrishnan, Phys. Rev. B 73 (2006) 035218.

[7] G.C. Milward, M.J. Calderón, P.B. Littlewood, Nature 433 (2005) 607-610.

[8] L.D. Landau, I.M. Lifshitz, Statistical Physics, Pergamon, Oxford, 1978 (section 139).

[9] C.H. Chen, S.-W. Cheong, Commensurate to incommensurate charge ordering and its real-space images in $\mathrm{La}_{0 .} \mathrm{Ca}_{0 .} \mathrm{MnO}_{3}$, Phys. Rev. Lett. 76 (1996) 4042

[10] S. Mori, T. Katsufuji, N. Yamamoto, C.H. Chen, S.-W. Cheong, Microstructure 
relatedto charge and orbital ordering in $\operatorname{Pr}_{0.5} \mathrm{Ca}_{0.5} \mathrm{MnO}_{3}$, Phys. Rev. B 59 (1999) 13573.

[11] R. Kajimoto, H. Yoshizawa, Y. Tomioka, Y. Tokura, Commensurate-incommensuratetransition in the melting process of orbital ordering in $\mathrm{Pr}_{0.5} \mathrm{Ca}_{0.5} \mathrm{MnO}_{3}$ : a neutron diffraction study, Phys. Rev. B 63 (2001) 212407.

[12] N. Furukawa, Y. Motome, cond-mat/0501738 unpublished and the experimental Refs. [23-26] therein.

[13] Y. Tokura, H. Kuwahara, Y. Moritomo, Y. Tomioka, A. Asamitsu, Phys. Rev. Lett. 76 (1996) 3184

[14] Y. Tokura, Y. Tomioka, J. Magn. Magn. Mater. 200 (1999) 1.

[15] E.O. Wollan, W.C. Koehler, Phys. Rev. 100 (1955) 545.

[16] J.B. Goodenough, Phys. Rev. 100 (1955) 564.

[17] F. Zhong, F.D. Wang, Phys. Rev. B 61 (2000) 3192.

[18] H. Kawano, R. Kajimoto, H. Yoshizawa, Y. Tomioka, H. Kuwahara, Y. Tokura, Phys. Rev. Lett. 78 (1997) 4253.

[19] H. Kawano-Furukawa, R. Kajimoto, H. Yoshizawa, Y. Tomioka, H. Kuwahara, Y. Tokura, Phys. Rev. B 67 (2003) 174422.
[20] F. Millange, S. de Brion, G. Chouteau, Phys. Rev. B 62 (2000) 5619.

[21] J. Garcia, M.C. Sanchez, G. Subias, J. Blasco, J. Phys.: Condens. Matter 13 (2001) 3229-3243.

[22] A. Daoud-Aladine, J. Rodríguez-Carvajal, L. Pinsard-Gaudart, M.T. FernándezDíaz, A. Revcolevschi, Phys. Rev. Lett. 89 (2002) 097205.

[23] M. Coey, Nature 430 (2004) 155.

[24] H.T. Stokes, D.M. Hatch, J.B. Campbell, ISOTROPY stokes.byu.edu/isotropy.html.

[25] D.V. Efremov, J. vandenBrink, D.I. Khomskii, Nat. Mater. 3 (2004) 853.

[26] Sang-Wook Cheong, Maxim Mostovoy, Nat. Mater. 6 (2007) 13.

[27] D.I. Khomskii, J. Magn. Magn. Mater. 306 (2006) 1.

[28] Y. Tokunaga, et al., Nat. Mater. 5 (2006) 937.

29] N. Biskup, A. de Andres, J.L. Martinez, C. Perca, Phys. Rev. B 72 (2005) 024115.

[30] A.M. Lopes, et al., Phys. Rev. Lett. 100 (2008) 155702.

[31] P.G. Radaelli, D.E. Cox, M. Marezio, S.W. Cheong, Phys. Rev. B 55 (1997) 3015.

[32] J.C. Tolédano, P. Tolédano, Landau Theory of Phase Transitions, Word Scientific, Singapore, 1988. 